\title{
AGENT BASED MANUFACTURING ${ }^{1}$
}

\author{
L. Monostori, J. Váncza \\ Computer and Automation Research Institute, \\ Hungarian Academy of Sciences, Budapest, Hungary; \\ Department of Production Informatics, Management and Control, \\ Budapest University of Technology and Economics, Budapest, Hungary \\ laszlo.monostori@sztaki.hu,vancza@sztaki.hu \\ Soundar Kumara \\ Department of Industrial and Manufacturing Engineering, \\ The Pennsylvania State University, University Park, PA 16802, USA \\ skumara@psu.edu
}

\begin{abstract}
Agent based manufacturing is an important area from a research and implementation points of view. In the past decade agent based manufacturing has become popular and many applications are built. In this keynote paper we survey the field with special emphasis on The International Institution of Production Research's activities in this area. The original extended paper is referenced at the end of this paper.
\end{abstract}

\section{EXTENDED ABSTRACT}

The emerging paradigm of agent-based computation has revolutionized the building of intelligent and decentralized systems. The new technologies met well the requirements in all domains of manufacturing where problems of uncertainty and temporal dynamics, information sharing and distributed operation, or coordination and cooperation of autonomous entities had to be tackled. In the paper software agents and multi-agent systems are introduced and through a comprehensive survey, their potential manufacturing applications are outlined. Special emphasis is laid on methodological issues and deployed industrial systems. After discussing open issues and strategic research directions, we conclude that the evolution of agent technologies and manufacturing will probably proceed hand in hand. The former can receive real challenges from the latter, which, in turn, will have more and more benefits in applying agent technologies, presumably together with well-established or emerging approaches of other disciplines.

The past decade has witnessed an explosive growth in computer, communication, and information technologies. High-performance computing, the world-wide web, universal access and connectivity, virtual reality, and enterprise integration are but a sampling of this revolution's many facets. At the same time, organizations and 
markets have also changed dramatically, represented by developments such as virtual organizations, business process integration, customer-centric supply chains, and electronic commerce. And, although industry strategists and academics continue to debate the precise future trajectory of changes in technologies and organizations, they agree that information - its availability, and the ability to exchange it seamlessly and process it quickly - lies at the core of organizations' abilities of meeting escalating customer expectations in global markets.

Agent-based computation is a new paradigm of information and communication technology that largely shapes and, at the same time, provides supporting technology to the above trends. Agent theories and applications have appeared in many scientific and engineering disciplines. Agents address autonomy and complexity: they are adaptive to changes and disruptions, exhibit intelligence and are distributed in nature. In this setting computation is a kind of social activity. Agents can help in self-recovery, and react to real-time perturbations. Agents are vital in the globalization context, as globalization refers to an inherently autonomous and distributed world both from geographical and information processing perspectives.

Agents - and similar concepts - were welcome in manufacturing because they helped to realize important properties as autonomy, responsiveness, redundancy, distributedness, and openness. Agents could be designed to work with uncertain and/or incomplete information and knowledge. Hence, many tasks related to manufacturing - from engineering design to supply chain management - could be conducted by agents, small and large, simple and sophisticated, fine- and coarsegrained that were enabled and empowered to communicate and cooperate with each other.

\footnotetext{
' The original version of this paper can be found in Annals of CIRP, Vol. 2, 2006
} 